\title{
Corela
}

Cognition, représentation, langage

HS-8 | 2010

L'interpellation

\section{Interpellation, dialogisme et mise en scène du discours narratif dans La chute d'A. Camus}

\section{Ligia Stela Florea}

\section{OpenEdition}

\section{Journals}

Édition électronique

URL : http://journals.openedition.org/corela/718

DOI : $10.4000 /$ corela. 718

ISSN : 1638-573X

\section{Éditeur}

Cercle linguistique du Centre et de I'Ouest - CerLICO

Référence électronique

Ligia Stela Florea, «Interpellation, dialogisme et mise en scène du discours narratif dans La chute d'A. Camus », Corela [En ligne], HS-8 | 2010, mis en ligne le 01 octobre 2010, consulté le 30 avril 2019. URL : http://journals.openedition.org/corela/718 ; DOI : 10.4000/corela.718

Ce document a été généré automatiquement le 30 avril 2019

\section{(c) (i) (2)(2)}

Corela - cognition, représentation, langage est mis à disposition selon les termes de la licence Creative Commons Attribution - Pas d'Utilisation Commerciale - Partage dans les Mêmes Conditions 4.0 International. 


\title{
Interpellation, dialogisme et mise en scène du discours narratif dans La chute d'A. Camus
}

\author{
Ligia Stela Florea
}

1 La présente contribution se propose d'examiner la fonction textuelle et discursive de l'interpellation dans le roman La chute d'A.Camus : son rôle dans la construction d'une figure d'allocutaire qui représente à la fois l'interlocuteur fictif du narrateur-personnage et une projection du lecteur.

2 On s'intéressera également aux rapports complexes que l'interpellation entretient avec le dialogisme, notamment avec la diaphonie, qui confère à l'allocutaire une présence effective dans la fiction (secondaire). Il y est présent non seulement à travers les diverses marques allocutives mais aussi par la manière dont se construit le discours qui lui est adressé.

3 Le fait que l'interpellation s'associe organiquement à un dialogisme marqué assigne au discours romanesque une structure d'échange (au sens de Roulet et alii 1985), inscrivant ainsi un discours à priori monologique dans une relation interlocutive et par là dans un espace interactif ayant son propre système de référence (cf. Vion 1992).

Dans la mesure où elle débouche sur une interaction verbale aplatie (car reposant essentiellement sur la diaphonie et sur le recipient designed) l'interpellation engendre une structure textuelle-discursive qui sert de déclencheur et de cadre scénographique (au sens de Maingueneau 2003), i.e. de mise en scène au discours narratif proprement dit.

5 Avant de passer à l'analyse de texte, on va procéder à une approche théorique portant sur les rapports de l'interpellation avec la fonction conative de Jakobson (1970), avec la catégorie d'intimation de Benveniste (1974) et avec le mode discursif allocutif de Charaudeau (1983). Cette démarche, qui nous fait passer d'une fonction du langage à un archétype discursif, nous amène inévitablement à l'une des questions centrales du colloque: l'interpellation est-elle ou non un acte de langage? 


\section{D'une fonction du langage à un archétype discursif}

\section{1 .}

6 Si le schéma de l'acte de communication, tel qu'il a été conçu par Jakobson en 1960, a fait l'objet de quelques sérieuses critiques, sa théorie des fonctions du langage garde encore son actualité, à en juger du moins d'après le rôle qu'on lui attribue dans les diverses typologies des textes ou des discours.

7 L'interpellation relève dans un premier temps de la fonction conative. Centrée sur le destinataire du message, celle-ci «trouve son expression grammaticale la plus pure dans le vocatif et l'impératif ", qui, sur le plan morphosyntaxique et même phonologique, «s'écartent des autres catégories nominales et verbales» (Jakobson 1970: 216). L'opposition que postulait Jakobson entre phrases impératives et phrases déclaratives préfigure et précède de près la première distinction de Austin entre énoncé performatif et énoncé constatif.

Dans la mesure où, comme on le verra plus loin, l'interpellation sert aussi à établir un contact avec un destinataire proche ou éloigné, elle nous semble participer aussi de la fonction phatique. Dans la pragmatique de l'interaction verbale on désigne sous le nom de phatiques les marqueurs discursifs qui assurent, en tandem avec les régulateurs, le maintien du contact avec l'autre et par là les mécanismes de «synchronisation interactionnelle » (cf. Kerbrat-Orecchioni 1990).

\section{2.}

9 Dans un article, considéré aujourd'hui comme le programme de la théorie de l'énonciation, Émile Benveniste (1974: 85) pose le « cadre figuratif de l'énonciation » qui fonde la structure du dialogue. Celle-ci suppose deux «figures en position de partenaires » qui assument alternativement le rôle de protagonistes de l'énonciation.

10 Parmi les grandes fonctions syntaxiques et discursives dont se sert l'énonciateur pour influencer de quelque manière le comportement de l'allocutaire, l'intimation occupe une place importante car elle suppose « un rapport vivant et immédiat de l'énonciateur à l'autre dans une référence nécessaire au temps de l'énonciation» (Benveniste 1974: 84). Quelle que soit la forme qu'elle revêt (ordre ou appel, impératif ou vocatif), l'intimation a partie liée avec l'interpellation.

\section{3 .}

11 Avec les « appareils langagiers » de Charaudeau (1983), on passe des fonctions du langage à ce que Bronckart appellera en 1993 « architypes discursifs ». Le modèle de Charaudeau comporte trois volets : énonciatif, argumentatif et narratif.

12 L'appareil énonciatif, où s'organisent les places et le statut des protagonistes et où se définissent leurs rapports et leur image de parole correspond au discours interactif de Bronckart. L'appareil argumentatif, où s'organise l'univers de discours en opérations de pensée de type cognitif correspond au discours expositif-théorique. L'appareil narratif, où 
s'organise l'univers de discours du point de vue des actions et des qualifications humaines renvoie chez Bronckart à deux architypes : le récit et la narration.

Le modèle de Charaudeau hérite aussi bien du modèle de Benveniste que de celui de Jakobson. Son appareil énonciatif renferme quatre composantes définies non comme un répertoire de marques linguistiques ou d'actes de parole mais «en termes de comportements langagiers » (Charaudeau $1983: 60$ ).

L'énonciatif polémique spécifie un comportement allocutif centré sur le sujet destinataire (TUd); l'énonciatif situationnel est un comportement élocutif centré sur le sujet énonciateur (JEé); l'énonciatif textuel et intertextuel dénotent tous les deux un comportement délocutif centré sur le propos énoncé (ILx). C'est l'énonciatif polémique qui nous intéresse pour l'étude de l'interpellation.

Charaudeau appelle cette composante " polémique " parce qu'elle dit quelque chose sur le rapport JE-TU (en prise directe l'un sur l'autre) mais aussi sur chacun de ces sujets en particulier : sur le TUd, qui se voit imposer l'univers de discours du JEé par le biais d'un contrat d'exécution, de même que sur le JEé auquel elle assigne une position d'autorité, qui varie selon le type de modalisation spécifiant ce comportement.

Les trois classes de modalisation qui spécifient le comportement allocutif sont:

- l'injonctif, qui définit le rapport JE-TU comme « comminatoire », c'est-à-dire qu'il donne au JEé un statut d'autorité absolue et au TUd un statut de soumission ;

- le discriminatif, qui définit le rapport JE-TU comme « interpellatif ", c'est-à-dire qu'il donne au JEé un statut d'autorité (le droit à interpeller) et au TUd un statut de sujet discriminé parmi un ensemble d'individus et désigné comme destinataire impliqué dans un rapport au JEé ;

- enfin le sollicitatif, qui définit le rapport JE-TU comme une « demande de dire ", c'est-à-dire qu'il donne au JEé le double statut de désir de savoir et d'autorité (le droit de questionner) et au TUd le double statut de possesseur du savoir et de soumission (obligation de répondre).

Ces modalisations peuvent se combiner entre elles pour donner naissance à différentes modalités illocutoires impliquant chacune un certain cadre énonciatif. Un seul et même acte peut donc combiner deux ou trois composantes de l'appareil énonciatif : l'ordre ou l'avertissement peuvent inclure un discriminatif et un injonctif alors qu'une demande d'information peut combiner un discriminatif et un sollicitatif.

Si le terme «interpellatif » sert ici à caractériser le rapport de places qui sous-tend la modalisation discriminative, les exemples illustrant cette modalisation combinent un appel et une injonction : Hép! Vous, là-bas ! ; S'il vous plaît, monsieur le Directeur !

\section{L'interpellation, acte de parole et unité conversationnelle}

\section{1.}

19 Nous partirons de cette conception de l'acte de parole pour distinguer:

- un sens restreint, où l'interpellation se définit comme un acte d'autorité, un sous-type d'acte directif associé à un contrat d'exécution ;

- un sens large où elle se définit comme une modalité d'établir un contact par la parole, une modalité discursive qui convertit un injonctif ou un sollicitatif en phatique. 
le Nouveau Petit Robert (2007), interpellation peut désigner depuis 1789 une demande d'explication adressée au gouvernement par un membre du Parlement en séance publique (ex.1) ou une sommation adressée par un agent de police à un passant lors d'un contrôle d'identité (ex.2). Sont à considérer également comme des actes d'autorité les appels par lesquels l'enseignant sollicite l'attention ou la participation des élèves, de même que les diverses injonctions servant de rappel à l'ordre (ex.3).

(1) Confirmez-vous, monsieur le Premier ministre qu'il ne s'agit pas d'un contrat à durée indéterminée mais d'un contrat à licenciement permanent?

(question concernant le CPE posée par le député socialiste Alain Vidalies dans la séance du 7 février 2006 de l'Assemblée Nationale, apud Sandré 2008)

(2) Hé, vous, là-bas, vos papiers s'il vous plaît! (exemple forgé)

(3) Attention tout le monde, c'est un jeu de mémoire!

Vous écoutez et moi je vous l'explique, hein!

Qui peut me dire comment ça se dit en anglais? Natacha! (exemples tirés de Rabatel 2004)

21 Au sens large, interpeller signifie «adresser brusquement la parole à quelqu'un pour interroger, insulter» (syn. apostropher, appeler)»(NPR 2007: 1357). Les exemples donnés par le NPR en guise d'illustration :

(4) Tout le monde se rencontrait, s'interpellait et conversait (Camus)

Les jeunes gens interpellant les filles... (Aragon).

Il s'agirait donc, selon cette acception, d'un ensemble d'actes qui combinent une apostrophe et une question ou une apostrophe et une invitation et qui servent à établir de manière intempestive un contact avec autrui, quel que soit le degré de connaissance ou les rapports affectifs et sans qu'intervienne un rapport d'autorité.

\section{2.}

Dans la perspective d'une approche interactionnelle, nous proposons de définir l'interpellation, à partir de cette deuxième acception, comme une unité conversationnelle, plus exactement comme une intervention initiative du type préséquence qui inclut, à part l'apostrophe, une demande d'information, de permission, d'excuse ou autre.

Selon la conception de Moeschler (1985: 98), qui s'inspire là de Shlegoff et de Levinson, la préséquence est une fonction interactive qui vise à «rendre acceptable, légitime interactionnellement et contextuellement l'acte directeur ». A la différence des préliminaires et des préparations, qui sont des fonctions orientées du point de vue illocutoire ou argumentatif, les préséquences ne donnent aucune indication sur la nature de l'acte directeur.

Ainsi, dans une intervention initiative comme celle que cite Moeschler : «Excusez-moi, monsieur. Est-ce que vous avez l'heure?» l'acte d'excuse ne ferait qu'annoncer une activité réparatrice sans spécifier la nature de l'acte réparateur. Son rôle est avant tout d'atténuer l'intrusion territoriale liée à l'énonciation de la requête.

Mais supposons que l'échange ne s'arrête pas une fois qu'il aura atteint la structure prototypique réparation/ satisfaction/ appréciation et qu'il se fasse suivre d'autres échanges, donnant lieu à tout un épisode conversationnel. Envisagée rétroactivement à ce niveau, l'intervention initiative citée par Moeschler acquiert dans sa totalité une fonction de préséquence. 
Lipit de La chute illustre parfaitement cette troisième acception du terme interpellation : «Puis-je, monsieur, vous proposer mes services sans risquer d'être importun?». C'est une interpellation au sens générique du terme: 'adresser brusquement la parole à quelqu'un pour interroger' (cf. Nouveau Petit Robert, 2007 : 1352).

Même si l'interrogation n'est ici qu'un acte primitif servant, en relation avec le verbe modal et le performatif, à construire un acte dérivé d'offre de service, elle reste une forme de discours intempestif qui abolit l'équilibre interactionnel et met en danger les faces des interlocuteurs.

29 Le protagoniste du roman, Jean-Baptiste Clamence, juge pénitent, vivant depuis dix ans à Amsterdam, propose à l'inconnu qu'il rencontre dans un bar de lui servir d'interprète auprès du gérant de l'établissement. Il interpelle son compatriote d'une manière aussi polie que possible, pour réduire les risques auxquels il s'expose :

(5) Puis-je, mo nsieur, vous proposer mes services sans risquer d'être importun? Je crains que vous ne sachiez vous faire entendre de l'estimable gorille qui préside aux destinées de cet établissement. Il ne parle, en effet, que le hollandais. A moins que vous ne m'autorisiez à plaider votre cause, il ne devinera pas que vous désirez du genièvre. Voilà, j'ose espérer qu'il m'a compris; ce hochement de tête doit signifier qu'il se rend à mes arguments. Il y va, en effet, il se hâte, avec une sage lenteur. Vous avez de la chance, il n'a pas grogné [...] Mais je me retire, monsieur, heureux de vous avoir obligé. Je vous remercie et j'accepterais si j'étais sûr de ne pas jouer les fâcheux. Vous êtes trop bon. J'installerai donc mon verre auprès du vôtre.

La préséquence d'interpellation correspond au premier énoncé et inclut une apostrophe et un acte d'offre associé à une demande de permission.

Il est intéressant de s'arrêter un peu sur les moyens dont se sert le locuteur pour parer aux effets négatifs de son interpellation: d'abord l'indirection (l'offre se joint à une demande de permission revêtant la forme d'une interrogation), puis le désarmeur sans risquer d'être importun, qui contient le présupposé "je ne veux pas être importun", ensuite la justification ( Je crains que vous ne sachiez...») suivie immédiatement d'une explication (« Il ne parle, en effet, que le hollandais»). Enfin, les formules de politesse : à commencer par l'appellatif déférent et les tournures au subjonctif qui relèvent d'un style châtié légèrement emphatique.

\section{3.}

Comme on peut le constater, l'interpellation est une modalité discursive qui va au-delà du simple appel à l'autre, englobant une requête et une demande d'excuse ou une requête et une justification. La préséquence qu'elle engendre est plus qu'une manière de s'adresser à l'autre pour lui demander quelque chose, c'est une manière d'entamer la conversation, d'établir une relation par la parole.

33 En effet, elle donne lieu dans la Chute à un long entretien qui montre que pour une nature communicative comme celle du protagoniste toute occasion de s'entretenir avec un « homme d'esprit » est une bonne aubaine. A en juger d'après les thèmes abordés, la conversation est éminemment orientée vers la construction d'une relation interpersonnelle.

Elle débute sous le signe de la gratuité absolue et glisse imperceptiblement du gérant du bar, affublé de l'aimable sobriquet de " gorille » à la ville d'Amsterdam et au caractère des Hollandais. Mais ce thème s'avère en fait un prétexte pour parler de Paris et de ses 
habitants. C'est là un objet de discours susceptible de rapprocher les deux personnages, même si le protagoniste adopte en parlant de ses compatriotes un ton d'ironie cinglante.

(6) Ferez-vous un long séjour à Amsterdam? Belle ville, n'est-ce pas? Fascinante? Voilà un adjectif que je n'ai pas entendu depuis longtemps. Depuis que j'ai quitté Paris, il y a des années de cela. [...] Paris est un vrai trompe-l'oeil, un superbe décor habité par quatre millions de silhouettes. Près de cinq millions, au dernier recensement? Allons, ils auront fait des petits. Je ne m'en étonnerai pas. Il m'a toujours semblé que nos concitoyens avaient deux fureurs: les idées et la fornication. A tort et à travers, pour ainsi dire. Gardons-nous, d'ailleurs, de les condamner; ils ne sont pas les seuls, toute l'Europe en est là [...]

Les Hollandais, oh non, ils sont beaucoup moins modernes. Que font-ils? Eh bien, ces messieurs-là vivent du travail de ces dames-là. Ce sont d'ailleurs, mâles et femelles, de fortes bourgeoises créatures [...] De temps en temps ces messieurs jouent du couteau ou du revolver, mais ne croyez pas qu'ils y tiennent. Le rôle l'exige, voilà tout...

L'échange suivant est celui des présentations; l'appellatif « docteur » qu'on vient de lui adresser est pour le protagoniste une occasion de dévoiler enfin son identité. Même si l'identité de l'autre se laisse attendre, c'est là un moment important pour la construction de la relation interpersonnelle. Tout en sachant qu'il convient de respecter la discrétion de son compatriote, Jean-Baptiste Clamence ne pourra pas s'empêcher de lui adresser deux questions des plus indiscrètes.

(7) On nous apporte enfin notre genièvre. A votre prospérité. Oui, le gorille a ouvert la bouche pour m'appeler docteur (...) Au demeurant, je ne suis pas médecin. Si vous voulez le savoir, j'étais avocat avant de venir ici. Maintenant, je suis jugepénitent.Mais permettez-moi de me présenter : Jean-Baptiste Clamence, pour vous servir. Heureux de vous connaître. Vous êtes sans doute dans les affaires? A peu près? Excellente réponse! Judicieuse aussi ; nous ne sommes qu'à peu près en toutes choses (...) Permettez-moi de vous poser deux questions et n'y répondez que si vous ne les jugez pas indiscrètes. Possédez-vous des richesses? Quelques-unes? Bon. Les avez-vous partagées avec les pauvres? Non. Vous êtes donc ce que j'appelle un saducéen. Si vous n'avez pas pratiqué les Ecritures, je reconnais que vous n'en serez pas plus avancé. Cela vous avance? Vous connaissez donc les Ecritures? Décidément, vous m'intéressez.

L'incipit du roman La chute sert à introduire, avant que ne se mette en place le discours diégétique, une figure d'allocutaire où il est impossible de ne pas entrevoir un avatar du lecteur lui-même. Le premier énoncé du texte peut très bien passer pour le discours interpellatif générique qu'un écrivain adresse d'emblée au lecteur en guise de captatio benevolentiae.

37 Ce discours construit aussi et surtout une figure de locuteur : l'ethos d'un homme habitué à manier les phrases, ethos d'un orateur au plein sens du terme, esprit cultivé et raffiné mais ironique et moqueur. L'autoironie est du reste la modalité indirecte que le protagoniste choisit dès le début pour se présenter à son interlocuteur : "A moins que vous ne m'autorisiez à plaider votre cause, il ne devinera pas que vous désirez du genièvre. Voilà [...] ce hochement de tête doit signifier qu'il se rendàmes arguments ». Les expressions en italique, de même que le style châtié et prétentieux jouent d'emblée un rôle décisif dans la construction de l'ethos. 


\section{Espace interactif et deixis fictionnelle}

\section{1.}

Mais une relation interlocutive est inséparable d'un contexte d'énonciation qui, bien que ne bénéficiant d'aucune description, est tout aussi présent dans le texte que les deux acteurs. Le contexte est introduit par un procédé du type showing, car il se construit par le biais de la référence associée au champ cognitif-perceptif du locuteur et de l'allocutaire.

Toute une série d'éléments concourent dans l'incipit du roman à mettre en place une situation dont les deux protagonistes sont partie prenante: la description définie l'estimable gorille, les déictiques spatiaux cet établissement, ce hochement de tête, voilà et les déictiques temporels : présent actuel (puis-je, je crains, vous désirez, j'ose espérer, il se rend, il $y$ va, il se hâte), passé composé (il m'a compris) et futur simple (il ne devinera pas).

40 À mesure que la conversation avance, le cadre situationnel se précise et s'élargit grâce la multiplication des déictiques personnels, spatiaux et temporels. Le moment où les deux compatriotes sont sur le point de se séparer, après avoir bu un second verre, mériterait lui aussi notre attention, car il s'amorce toujours par une interpellation :

(8) Vous partez déjà ? Pardonnez-moi de vous avoir peut-être retenu. Avec votre permission, vous ne paierez pas. Vous êtes chez moi à Mexico-City, j'ai été particulièrement heureux de vous y accueillir. Je serai certainement ici demain, comme les autres soirs et j'accepterai avec reconnaissance votre invitation. Votre chemin... Eh bien...Mais verriez-vous un inconvénient, ce serait le plus simple, à ce que je vous accompagne jusqu'au port? De là, en contournant le quartier juif, vous trouverez ces belles avenues où défilent des tramways chargés de fleurs et de musiques tonitruantes. Votre hôtel est sur l'une d'elles, le Damrak. Après vous, je vous en prie.

Il est évident que la question de J.B.Clamence enchaîne sur du non verbal: son interlocuteur s'apprête à partir, peut-être qu'il se lève déjà.

La demande d'excuse se fait suivre d'un acte complexe qui ne fait que rééditer l'interpellation du début: offre assortie d'une demande de permission. En échange, la promesse je serai certainement ici demain, enchaîne selon toute évidence sur une intervention implicite de l'interlocuteur qui voulait savoir quand il pourrait prendre sa revanche.

Le fragment d'énoncé votre chemin annonce un changement de décor dont les grandes lignes se dessinent déjà grâce aux descriptions définies le port, le quartier juif, ces belles avenues, le Damrak. L'énoncé inachevé reprend en écho une demande d'information, qui suscite de la part du narrateur une nouvelle offre associée à une demande (indirecte) de permission : « mais verriez-vous un inconvénient à ce que je vous accompagne... ?»

Mais est ici non seulement un inverseur argumentatif signalant que l'énoncé qu'il introduit s'oppose aux conclusions qu'on pourrait retirer de votre chemin...eh bien où se joignent la fin d'une question et l'amorce d'une réponse. Mais est aussi un connecteur pragmatique reliant par-delà les actes de parole deux comportements opposés du narrateur : l'intention de quitter l'étranger, après l'avoir renseigné sur son chemin, et l'offre de l'accompagner jusqu'au port et de continuer la conversation. C'est un mais analogue à celui qu'il avait utilisé au début pour annoncer son intention de se retirer, contredite peu après par la décision d'accepter l'invitation de l'autre. 


\section{2.} discours romanesque - traditionnellement monologique - une structure d'échange.

La construction du site, qu'il s'agisse du Mexico-City, des rues d'Amsterdam, de l'île de Marken, du lac de Zuyderzee ou, pour finir, de la chambre même du protagoniste, se réalise par des actes de référence composant et simulant a first person thinking reference (cf. Castañeda 1989). L'univers référentiel associé à l'espace interactif s'organise en fonction du champ cognitif et perceptif des deux protagonistes; plus exactement, les actes de référence prennent pour origine le ToSo de la situation d'énonciation représentée par le texte, de sorte que la perspective du locuteur devient la perspective commune des deux participants.

On voit ainsi se mettre en place une deixis fictionnelle, i.e. une deixis de la fiction secondaire, ancrée dans la temporalité de l'acte de narration. C'est le système de référence que se donne la scène d'énonciation narrative (cadre scénographique) construite par le texte (cf. Maingueneau 1987 et 2003). C'est une deixis empathique ancrée dans un éternel présent qui s'actualise à travers l'acte de lecture. Par le biais du contrat de fiction, le lecteur se voit imposer ainsi l'univers de discours et l'univers référentiel du narrateur, qu'il finit par intégrer par un mécanisme d'exotopie réceptive (cf. Bakhtine 1978).

Le passage le plus intéressant à cet égard nous semble le voyage à l'île de Marken et la description du Zuyderzee.

(9) Un village de poupée, ne trouvez-vous pas? Le pittoresque ne lui a pas été épargné! Mais je ne vous ai pas conduit dans cette île pour le pittoresque, cher ami. Tout le monde peut vous faire admirer des coiffes, des sabots [...] Je suis un des rares à pouvoir vous montrer ce qu'il y a d'important ici.

Nous atteignons la digue. Il faut la suivre pour être aussi loin que possible de ces trop gracieuses maisons. Asseyons-nous, je vous en prie. Qu'en dites-vous? Voilà, n'est-ce pas, le plus beau des paysages négatifs. Voyez, à notre gauche, ce tas de cendres qu'on appelle ici une dune, la digue grise à notre droite, la grève livide à nos pieds et, devant nous, la mer couleur de lessive faible, le vaste ciel où se reflètent les eaux blêmes [...] N'est-ce pas l'effacement universel, le néant sensible aux yeux? Pas d'hommes, surtout pas d'hommes! Vous et moi, seulement, devant la planète enfin déserte!

On a là une illustration des plus spectaculaires de la mise en scène du discours narratif.

L'interpellation s'associe à un mode discursif allocutif contenant des demandes de dire, notamment des demandes de confirmation (ne trouvez-vous pas, n'est-ce pas) mais aussi des demandes de faire : asseyons-nous, voyez. La deixis empathique repose ici sur trois séries d'éléments : thématisation d'une entité non verbale (image visuelle), recours au PR actuel et aux marques indexicales (descriptions définies, SN démonstratifs, déictiques spatiaux).

Ce genre de séquences descriptives construit ainsi ce qu'on pourrait appeler un point de vue partagé, c'est-à-dire une perception/pensée attribuée au couple narrateur/ narrataire, contribuant de façon décisive à activer les mécanismes d'identification empathique du lecteur avec le narrataire.

\section{Inscription du dialogue dans le discours monologal}

51 L'effet le plus intéressant de l'interpellation est le dialogisme marqué, qui imprime au 
52 Selon Bakhtine (1977, 1978), tout discours, même monologal, présente un dialogisme constitutif qu'il appelle dialogisation interne. L'originalité de ce discours romanesque réside en ce qu'il force les limites de la dialogisation interne jusqu'au point où elle côtoie la dialogisation externe. La chute construit un discours monologal qui ne se borne pas à réagir à un discours étranger sur le mode de la confirmation ou de la réfutation.

53 Tantôt il s'applique à reconstruire le discours étranger par des reprises diaphoniques (dialogisme interdiscursif), tantôt il entreprend de le provoquer par des interpellations, des injonctions ou des questions (dialogisme interlocutif, cf. Bakhtine 1978). A ces procédés s'ajoutent des marqueurs typiquement dialogaux : des phatiques (allons, tenez, voyons, n'est-cepas, voyez-vous, voulez-vous) et des régulateurs (oui, bon bon, vous avez raison). Le discours monologal arrive ainsi à mettre en scène un dialogue.

En effet, les trois premières pages du roman se laissent diviser en plusieurs séquences comportant chacune une structure d'échange. Dans les termes de Roulet et alii (1985), les constituants immédiats qui se dégagent du texte sont des interventions constitutives d'échange liées par des fonctions illocutoires initiative et réactive, ce qui permet de procéder à un découpage dialogal.

(5')L : Puis-je, monsieur, vous proposer mes services sans risquer d'être importun? Je crains que vous ne sachiez vous faire entendre de l'estimable gorille qui préside aux destinées de cet établissement.

A : Il ne parle pas français?

$\mathrm{L}:$ Il ne parle, en effet, que le hollandais. A moins que vous ne m'autorisiez à plaider votre cause, il ne devinera pas que vous désirez du genièvre. Voilà, j'ose espérer qu'il m'a compris; ce hochement de tête doit signifier qu'il se rend à mes arguments [...] Mais je me retire, monsieur, heureux de vous avoir obligé. A : Venez vous installer là, si vous voulez!

$\mathrm{L}$ : Je vous remercie et j'accepterais si j'étais sûr de ne pas jouer les fâcheux.

A : Vous ne m'importunez pas, au contraire.

$\mathrm{L}$ : Vous êtes trop bon. J'installerai donc mon verre auprès du vôtre.

(6')

L : Ferez-vous un long séjour à Amsterdam? Belle ville, n'est-ce pas?

A : Fascinante

L : Voilà un adjectif que je n'ai pas entendu depuis longtemps. Depuis que j'ai quitté Paris, il y a des années de cela. [...] Paris est un vrai trompe-l'oeil, un superbe décor habité par quatre millions de silhouettes.

$\mathrm{A}$ : Près de cinq millions, au dernier recensement.

L : Allons, ils auront fait des petits. Je ne m'en étonnerai pas. Il m'a toujours semblé que nos concitoyens avaient deux fureurs: les idées et la fornication. A tort et à travers, pour ainsi dire. Gardons-nous, d'ailleurs, de les condamner; ils ne sont pas les seuls, toute l'Europe en est là [...]A : Les Hollandais aussi ?

L : Les Hollandais, oh non, ils sont beaucoup moins modernes.

A : Que font-ils?

L : Eh bien, ces messieurs-là vivent du travail de ces dames-là. Ce sont d'ailleurs, mâles et femelles, de fortes bourgeoises créatures [...] De temps en temps ces messieurs jouent du couteau ou du revolver, mais ne croyez pas qu'ils y tiennent. Le rôle l'exige, voilà tout [...] Ceci dit, je les trouve plus moraux que les autres, ceux qui tuent en famille, à l'usure. N'avez-vous pas remarqué que notre société s'est organisée pour ce genre de liquidation?

(7')L : On nous apporte enfin notre genièvre. A votre prospérité.

A : Le gorille a ouvert la bouche pour vous appeler docteur.

L: Oui [...] Au demeurant, je ne suis pas médecin. Si vous voulez le savoir, j'étais avocat avant de venir ici. Maintenant, je suis juge-pénitent. Mais permettez-moi de me présenter: Jean-Baptiste Clamence, pour vous servir. Heureux de vous connaître. Vous êtes sans doute dans les affaires? 
$A$ : A peu près.

$\mathrm{L}$ : Excellente réponse! Judicieuse aussi; nous ne sommes qu'à peu près en toutes choses [...] Permettez-moi de vous poser deux questions et n'y répondez que si vous ne les jugez pas indiscrètes. Possédez-vous des richesses?A: Quelques-unes.

$\mathrm{L}:$ Bon. Les avez-vous partagées avec les pauvres?

A : Non.

L : Vous êtes donc ce que j'appelle un saducéen. Si vous n'avez pas pratiqué les Ecritures, je reconnais que vous n'en serez pas plus avancé.

A : Mais si, cela m'avance.

L : Vous connaissez donc les Ecritures? Décidément, vous m'intéressez.

Chaque séquence s'amorce par une interpellation (offre de service, question, vœu) qui suscite à chaque fois chez l'allocutaire une réaction verbale ou non verbale dont le discours du narrateur fixe la trace. La diaphonie consiste, selon Roulet et alii (1985: 71) à reprendre et à "réinterpréter dans son propre discours la parole du destinataire pour mieux enchaîner sur celle-ci ».

Il convient de distinguer ici reprises diaphoniques et réactions à un discours implicite. Les reprises, qui sont de loin la stratégie dominante, sont repérables dans les trois séquences : en (5) «il ne parle en effet que le hollandais », en (6) "fascinante », "que font-ils » et surtout en (7) «à peu près", "quelques-unes", "non", "cela vous avance». Les réactions à un discours implicite se réduisent à deux occurrences : « je vous remercie » et " vous êtes trop bon », toutes les deux dans la séquence (5).

Ces procédés concourent non seulement à simuler un dialogue parfaitement cohérent mais aussi à susciter et à relancer le discours narratif. Le dialogisme marqué intervient de manière décisive dans la construction d'un espace interactif fictionnel qui sert de déclencheur et de dispositif scénique au discours narratif.

\section{L'interpellation comme stratégie discursive}

On a pu constater que l'interpellation joue un rôle important dans la construction d'une figure de locuteur et d'allocutaire, dans la mise en place d'une relation interlocutive inséparable d'un référentiel énonciatif et par-dessus tout dans la mise en place d'un discours dont les structures dialogiques frisent de près les structures dialogales.

Ce qui amène à conclure que l'interpellation et le mode discursif allocutif qu'elle engendre ont dans La chute d'A.Camus une fonction structurante. D'une part ils mettent en place un dispositif textuel-discursif servant de déclencheur et de cadre scénographique au discours narratif proprement dit. D'autre part, ils servent à relancer le discours narratif d'une séquence à l'autre et d'un chapitre à l'autre contribuant ainsi à ponctuer et à structurer le texte du roman.

La fonction structurante de cette stratégie discursive joue selon nous à trois niveaux :

- le mode allocutif avec tout ce qu'il suppose (adresse directe, actualisation de la relation interlocutive, référence au contexte, simulation de l'échange) a le rôle de marquer le changement de plan discursif : de la fiction secondaire à la fiction principale et vice versa ;

- au cadre de la fiction secondaire, chaque préséquence interpellative a le rôle de préparer et de légitimer la poursuite du récit-confession, qui est une activité à risque. Les préséquences, le mode allocutif et la dialogisation sont des stratégies destinées à favoriser l'entrée en confidence et le déploiement de cette activité discursive ; 
- la préséquence interpellative et le mode allocutif jouent un rôle important dans l'organisation même du texte : dans la division et le marquage des épisodes et du plan global de texte. Pour s'en convaincre, il suffit d'examiner brièvement les incipits de chaque unité textuelle (ou chapitre).

Chaque chapitre s'ouvre par une interpellation, à l'exception du dernier, qui s'achève par une telle séquence. On va les reprendre chacun par leur numéro.

1. Début: Puis-je, monsieur, vous proposer mes services sans risquer d'être importun? Je crains que vous ne sachiez vous faire entendre de l'estimable gorille qui préside aux destinées de cet établissement.

2. Début : Qu'est-ce qu'un juge pénitent ? Ah! je vous ai intrigué avec cette histoire. Je n'y mettais aucune malice, croyez-le, et je peux m'expliquer plus clairement. l'inconnu qui vient de franchir le seuil du Mexico-City, le second chapitre commence par une question. Le cotexte nous dit qu'il s'agit d'une reprise en écho et que par conséquent la question doit être attribuée au co-énonciateur.

Ce qu'elle a d'intéressant une telle interpellation, c'est que, dans la perspective de l'acte de production (inscrit dans la fiction secondaire), elle se présente comme issue de la rencontre avec un discours antérieur, donc comme relevant du dialogisme interdiscursif. Dans la perspective de l'acte de réception (lecture), la préséquence interpellative relève au contraire du dialogisme interlocutif, car elle anticipe sur une réaction possible du lecteur.

La séquence allocutive qui suit annonce clairement l'intention de fournir l'explication sollicitée et précède de près la séquence narrative, qui s’amorce dès le second alinéa : « Il y a quelques années, j'étais avocat à Paris et, ma foi, un avocat assez connu ».

3. Début: Vraiment, mon cher compatriote, je vous suis reconnaissant de votre curiosité. Pourtant, mon histoire n'a rien d'extraordinaire. Sachez, puisque vous y tenez, que j'ai pensé un peu à ce rire, pendant quelques jours, puis je l'ai oublié.

Le troisième chapitre s'ouvre par un remerciement. On n'a plus affaire à une reprise diaphonique mais à une reprise par implicitation : le remerciement ne fait que réagir à un discours antérieur, dans ce cas une question ou une injonction qui encourageait et engageait le protagoniste à poursuivre son histoire.

66 La suite du texte le confirme : «Sachez, puisque vous y tenez, que j'ai pensé un peu à ce rire...». Cet énoncé métadiscursif sert à introduire dès le premier alinéa un nouvel épisode de l'histoire : l'épisode clef du suicide de la jeune fille.

4. Début : Un village de poupée, ne trouvez-vous pas? [...] Mais je ne vous ai pas conduit dans cette île pour le pittoresque, cher ami. Tout le monde peut vous faire admirer des coiffes, des sabots... Je suis un des rares à pouvoir vous montrer ce qu'il y a d'important ici.

Le quatrième chapitre s'ouvre par une demande de confirmation, ou, dans les termes de l'analyse conversationnelle, par un marqueur de recherche d'approbation discursive.

68 Sa présence au début du chapitre qui retrace le voyage à l'île de Marken n'est pas du tout accidentelle. Tout comme la description in situ qu'elle introduit (voir supra 3), la demande de confirmation assume une fonction importante du point de vue interactionnel : activer et stimuler le partage des représentations et des émotions.

69 A l'échelle du discours romanesque, la construction d'un point de vue partagé est une stratégie servant à la mise en scène du discours narratif et à l'activation des mécanismes d'identification empathique du lecteur avec le narrataire.

Corela, HS-8 | 2010 
5. Début: Vous vous trompez, cher, le bateau file à bonne allure. Mais le Zuyderzee est une mer morte ou presque [...] Alors nous marchons sans aucun repère, nous ne pouvons évaluer notre vitesse. Nous avançons et rien ne change [...] Dans l'archipel grec j'avais l'impression contraire.

Le cinquième chapitre commence par un acte de réfutation qui renvoie à une intervention implicite de l'allocutaire, relativement facile à restituer: "On dirait que le bateau n'avance pas ». Ce début de chapitre est symétrique au précédent, car il sert à embrayer le discours sur une nouvelle description. Comme l'archipel grec n'est ici qu'un souvenir, ce qu'on va nous présenter cette fois n'est plus un décor in situ mais un décor remémoré.

Il convient de s'arrêter un moment sur le terme d'adresse, cher qui marque une nette progression par rapport à l'appellatif monsieur du début du roman. Les termes d'adresse indiquent dans leur succession une réduction significative de la distance interpersonnelle : de monsieur, chercompatriote à cher ami, cher et cher maître.

6. Fin : Alors, racontez-moi, je vous prie, ce qui vous est arrivé un soir sur les quais de la Seine...

Dans le dernier chapitre la séquence interpellative est située à la fin : elle clôt ainsi le chapitre et le roman qui s'achève à peu près comme il avait commencé.

Mais cette position n'enlève pas à l'interpellation sa fonction de préséquence, car la prière que Jean-Baptiste Clamence adresse à son interlocuteur ne fait qu'annoncer un autre entretien, un autre récit et une autre confession.

74 L'interpellation finale s'associe dans l'économie du roman à un déclencheur 2, c'est-à-dire au point culminant : le moment où l'étranger dévoile enfin son identité et où l'on se rend compte que cet autre est en fait un alter-ego, une instance qui consacre, par-delà la complicité entre narrateur et narrataire, celle de l'écrivain et de son lecteur: " Hypocrite lecteur - mon semblable - mon frère ! »

\section{BIBLIOGRAPHIE}

Bakhtine, M. (1977), Le marxisme et la philosophie du langage, Paris, Editions de Minuit.

Bakhtine, M. (1978), Esthétique et théorie du roman, Paris, Gallimard.

Benveniste, E. (1974), Problèmes de linguistique générale, 2, chapitre V, Paris, Gallimard.

Bres, J. (1998), « Entendre des voix : de quelques marqueurs dialogiques en français », J.Bres, R.Legrand, F.Madray et P.Siblot (éds), L'autre en discours, Montpellier III, Praxiling, p.191-212.

Bronckart, J.P. (1993), « L'organisation temporelle des discours. Une approche de psychologie du langage », Langue française, 97, p.3-13.

Camus, A. (1956), La chute, Paris, Gallimard, collection Folio.

Castañeda, H.-N. (1989), Thinking, Language and Experience, University of Minnesota Press.

Charaudeau, P. (1983), Langage et discours. Eléments de sémiolinguistique, Paris Hachette. 
Ducrot, O. et alii (1980), Les mots du discours, Paris, Editions de Minuit.

Ducrot, O. (1984), Le dire et le dit, Paris, Editions de Minuit.

Florea, L.S. (1993) «Injonctif vs optatif. Analyse des rapports entre mode et modalité en français et en roumain ", Actes du XX-e Congrès de Linguistique et de Philologie romanes - Zürich 1992, tome I, Francke Verlag, Tübingen und Basel, p.219-234.

Florea, L.S. (2005), « Narration au présent, deixis fictionnelle et point de vue », Revue de sémantique et pragmatique, 17, 69-88.

Jakobson, R. (1970), Linguistique et poétique, in Essais de linguistique générale, Paris, Seuil.

Kerbrat-Orecchioni, C. (1990 et 1992), Les interactions verbales, vol. I et II, Paris, A.Colin.

Maingueneau, D. (1986/2003), Linguistique pour le texte littéraire, 4e édition, Paris, Nathan.

Maingueneau, D. (1987), Nouvelles tendances en analyse du discours, Paris, Hachette.

Moeschler, J. (1985), Argumentation et conversation, Paris, Hatier-Crédif.

Rabatel, A., dir. (2004), Interactions orales en contexte didactique, IUFM de l'Académie de Lyon, Presses univesitaires de Lyon.

Roulet, E. et alii (1985), L'articulation du discours en français contemporain, Berne-New York-Paris, Peter Lang.

Roulet, E. (1999), La description de l'organisation du discours, ENS de Fontenay/Sant-Cloud, Didier, Collection LAL.

Sandré, M. (2008), « Les mésaventures de l'interaction : l'interruption, signe d'une difficulté interactionnelle ou mécanisme de régulation? ", Actes du XXVe CILPR, Innsbruck, 2007 (à paraître).

Vion, R. (1992), La communication verbale. Analyse des interactions, Paris, Hachette Université : Linguistique.

Vuillaume, M. (1990), Grammaire temporelle des récits, Paris, Editions de Minuit.

\section{AUTEUR}

\section{LIGIA STELA FLOREA}

Université Babeş-BolyaiCluj-Napoca 